\title{
A Prompt Response to COVID-19 Pandemic 2020: Delivery of Medications for Care Recipients of NCD Clinics at National Hospital Kandy, Sri Lanka.
}

T.M.T.A. Tennakoon ${ }^{a}$ (i) W.M.A. Wijekoon ${ }^{b}$ (i),P.E.K.B. Ranatungac ${ }^{(0)}$, R.M.S.K. Ratnayake ${ }^{a}$ (i) Charles Antonypillai ${ }^{(10}$, I.W.Y.K. Rambukwella ${ }^{a}$ (i)

\section{ABSTRACT}

The Government of Sri Lanka realised the possibility of COVID-19 becoming a pandemic and its probability of reaching Sri Lanka, even before the World Health Organisation declared COVID-19 as a pandemic. GOSL adopted a strategy focusing on minimising the risk of entry, containment, flattening the curve and minimising fatalities.

To ensure social distancing for persons with chronic illnesses, a guideline was issued to deliver the medications to the doorstep of the care recipients who attend the government sector hospital clinics for Non-Communicable Diseases. To facilitate the delivery of medications, National Hospital, Kandy decided to use Patient Information Management systems available at the hospital.

The care recipients were contacted over the phone, and their data was taken and fed into the electronic systems. Medical officers entered relevant prescriptions to the system, and then electronic prescriptions were used by the pharmacy for dispensing medications. Insulin was delivered to care recipients through primary and secondary hospitals of the Department of Health Services of Central Province with the help of Regional Director of Health Services Kandy. All other medications were seal packed and delivered via the Department of Posts - Sri Lanka.

There were several challenges, including staff training, process flow issues, the inadequacy of resources and change management concerns regarding the procedure. Despite the challenges, $90 \%$ of care recipients of Non-Communicable Diseases clinics were registered to the system, electronic prescriptions were issued, and medications were delivered to them through the government postal service. This demonstrated that during COVID-19 pandemic, institutions under three different government sector organisations in the Central Province were able to collaborate and efficiently adapt to the urgent, unexpected situations.

Keywords: COVID-19, pandemic, Non-Communicable Diseases, clinic care recipients, Digital Health, Sri Lanka.

\section{INTRODUCTION}

The World Health Organisation (WHO) on 30th January 2020, announced that the Coronavirus Disease 2019 (COVID-19) as a public health emergency of international concern. On 11th March 2020, it was declared as a pandemic by the WHO(1).

The Severe Acute Respiratory Syndrome Coronavirus 2 (SARS-CoV-2) transmits human-tohuman primarily during close contact via respiratory droplets produced by coughing or sneezing. It also transmits indirectly via contaminated surfaces. Since the incubation period is ranging from five to 14 days, and the majority of infected are asymptomatic, or with mild symptoms, the risk of transmitting the virus by a healthy person is high ${ }^{(2)}$.

No vaccination has been approved for full use for the disease to date. The primary intervention strategy is to flatten the epidemic curve by social distancing and isolation of

(C) This is an open access article licensed under a Creative Commons Attribution-Share Alike 4.0 International License (CC BYSA 4.0), which permits unrestricted use, distribution and reproduction in any medium, provided the original author and source are attributed and materials are shared under the same license.

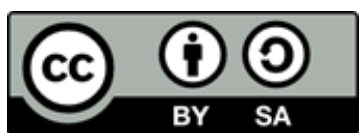


suspected contacts.

On 27th January, the first confirmed case of the COVID-19, a 44-year-old female from Hubei Province in China on a leisure tour, was reported in Sri Lanka. On 10th March, the first Sri Lankan was tested positive for COVID-19(3). The Government of Sri Lanka (GOSL) imposed a nation-wide police curfew on 20th March to ensure social distancing. To reduce the transmission of the virus, social distancing was encouraged by the Ministry of Health (MoH) guidelines(4). The guidelines issued on this regard recommended reducing non-urgent, in-person hospital visits and elective procedures, including routine surgeries. internet, video conferencing and satellite-based communications. Telehealth includes both preventive and curative aspects of healthcare delivery. Telemedicine on the other hand is the curative or the clinical part of Telehealth ${ }^{(5,6)}$ and can be described as the process of exchanging medical information from one site to another via electronic communications to improve a patient's clinical health status'(7). The types of information that can be transferred from site to site include clinical documents, laboratory results, digital photographs, ECGs, radiographs, MRI scans, CT scans, real-time ultrasonography, video recordings, auscultatory sound from electronic stethoscopes and physiological examination parameters such as blood pressure, heart rate, haemoglobin saturation, spirometry, etc.

\section{CASE DESCRIPTION}

The case site National Hospital of Kandy (NHK) is a 2629 bedded tertiary care hospital in the city of Kandy. NHK, the 2nd largest hospital in Sri Lanka, is directly managed under the $\mathrm{MoH}$. The hospital caters for nearly 45,000 NonCommunicable Disease (NCD) clinic care recipients per month ${ }^{(8)}$. The clinic care recipients visit the hospital regularly and are seen by a medical officer, and the medications were delivered to hand by the pharmacy.

Situated in the Kandy district of the Central Province of Sri Lanka, NHK is the main tertiary care hospital for the province. Though the hospital caters without a strict catchment area boundary, the majority of the care recipients are resident within the province.

When the GOSL enforced lockdowns through police curfew, the routine hospital clinic system of the NHK was affected as the care recipients were no longer allowed to visit the clinics physically. In keeping with the policy of social distancing, the $\mathrm{MoH}$ decided to deliver the medications to the doorstep, while ensuring a regular supply of medication to the care recipients ${ }^{(9)}$. In compliance with all these recommendations and limitations, the NHK decided to restrict all the routine clinic follow-ups and to dispatch clinic medication for the upcoming months through the Department of Posts - Sri Lanka.

During the deliberations that followed, specific practical issues were identified that needed immediate attention for the successful execution of this process.

1. Most of the postal addresses of clinic care recipients in the hospital records were vague or incorrect and, in some cases, were not available at all. Even the contact phone numbers of most of the care recipients were not available in clinic records

2. Cost of delivery of medications

3. Maintenance of cold chain, during the delivery of medication

4. Requirement of performing examinations and investigations prior to issuing prescriptions.

\section{OBJECTIVES}

The main objective was to deliver the medications to NCD clinic care recipients during the pandemic to ensure social distancing and safety of the care recipients. Rapid implementation of the clinic module of Patient Information Management (PIM) systems and making the clinic staff familiarise with PIM systems in order to expedite clinic activities of NHK were among primary targets to achieve the above objective. Continuing the same process after the health situation of the country returns to normalcy, was recognised as a long-term objective.

\section{THE APPROACH}

A quick plan was developed to address the identified practical issues.

The Hospital Information Management System (HIMS), the adapted version of electronic medical record by the $\mathrm{MoH}$, was already in operation at NHK since January 2017(10). There was another Clinic Management System (CMS) implemented at Diabetes and Endocrinology (D\&E) clinic since October 2019. It was decided to implement clinic and pharmacy modules of those two PIM systems for the management of other NCD clinic care recipients' data to facilitate the delivery of medication to them.

An assessment of the current ICT infrastructure of NHK revealed the need for suitable new hardware and further staff training. The hospital had two server computers, and most of the clinics already had necessary Local Area 
Networks (LAN). Therefore, the general clinic module, along with the pharmacy module could be implemented at this time.

A plan was drafted initially concerning the clinic medication dispensation. According to that plan, the following steps were taken.

- $\quad$ All the NCD clinics in the hospital were identified and enlisted.

- A questionnaire was sent to each specialist medical officer inquiring whether they had contact details of the care recipients and prescriptions for them.

- It was decided that each unit was responsible for contacting care recipients, getting their details and entering care recipient data to the system.

- Prescriptions to be entered into the system by the respective medical officers.

- $\quad$ The Health Information and Research Unit (HIRU) should facilitate and coordinate the process (provision of the necessary hardware, software, network connections, phone lines, training and finding volunteers).

- In respect of care recipients whose contact details were not available, their attention will be drawn through mass media and a request will be made to contact the hospital and give their details to the direct contact numbers of the clinics.

An internal circular was issued for NCD clinics along with the questionnaire. Several direct phone lines were established for each clinic. Each care recipient was contacted, and details required for the registration at PIM systems were acquired by the nursing staff of the NCD clinics and volunteers, who were specially recruited for this purpose. Care recipients also could give their details through the published direct phone lines. Once the care recipient data was available at hand, registration of care recipients in PIM systems along with the clinic admissions commenced. A Personal Health Number (PHN) that is being introduced as the unique identifier of healthcare recipients of Sri Lanka was generated for each care recipient who did not have a PHN through HIMS(11).

Once care recipients were registered in the relevant clinic with their demographic data, medical officers in the respective clinics started to enter their prescriptions to the respective system. The medication list was loaded into the electronic system with available strengths, routes, most common frequencies and duration; thus, only the selection of medication from a list could be done, and typing was minimal. Due to the above practice, the errors were minimised, and the process became efficient. The pharmacist could view and print the electronic prescription entered by the medical officer and dispense the medications accordingly. The pharmacists were then instructed to issue and pack the medications and hand them over to the Department of Posts - Sri Lanka, to be sent to the care recipients.

The Department of Posts operated at their full capacity to deliver the medications. They could utilise their existing human and physical resources without restrictions as other regular postal services were suspended at the time, due to safety concerns. They assured that the care recipients receive the correct medication, and if there was any discrepancy in the name or address, medication was returned to the hospital.

However, insulin could not be sent through the post as it required optimum temperatures maintenance during transport. As insulin was frequently used for patients suffering from Diabetes Mellitus, it was essential that the drug was transported under cold chain requirements to the care recipient. The Department of Health Services of Central Province (DoHS-CP) agreed to distribute it with the help of RDHS Kandy. DoHS-CP has an extended curative care network through 158 hospitals and had facilities to transport drugs which required temperature control.

NCD clinic care recipients in need of essential investigations or had complaints about unusual or new symptoms, were advised to visit the hospital.

\section{THE OUTCOMES}

In accordance with social distancing guidelines and recommendations to reduce in-person routine clinic visits, NHK was able to execute its plan and put in place the PIM systems to facilitate smooth delivery of medications to the care recipients. Care recipient registration to PIM systems was started on 29th March 2020. Since the clinic and pharmacy modules for HIMS were already developed and tested, it consumed little time to implement it fully at NHK. Initially, it was implemented at the Cardiology clinic, and the first electronic prescription was issued on 6th April 2020. Since CMS of D\&E clinic has already been implemented, they continued with patient registration and issuance of prescriptions. The HIMS clinic module was implemented in other clinics. The HIMS generated PHN, which is a unique value for each patient, facilitated the identification of patients. When a care recipient visits more than one clinic, he is registered with the same PHN but with different clinic numbers unique to each clinic. 
This facilitated the process of generating prescriptions and packing and posting of medications without hassle. During the phase of contacting care recipients over the phone and registering them to PIM systems, the contribution of volunteers was remarkable.

At the end of two months, 40,150 NCD clinic care recipients of 12 NCD clinics were registered in the system, 46,650 electronic prescriptions were issued, and medications delivered to clinic care recipients through the post.

\section{THE CHALLENGES AND THE STRATEGIES ADOPTED}

Since the NHK addresses a diverse set of patient care and operational needs at several clinics, the significance of PIM systems for social distancing became apparent during the pandemic. An assessment of current requirements was carried out to determine the operational procedures required for pandemic management within the clinics, the existing information structure to support these processes, and the gaps that needed to be bridged promptly. It necessitated configuring PIM systems to support COVID19 pandemic management at NHK clinics.

The advice of the Ministry of Health, Sri Lanka concerning the medications of care recipients, was to send them through post after contacting them over the phone and getting their addresses confirmed. Every effort was taken to enhance informatics infrastructure while facilitating the medication delivery to clinic care recipients during the pandemic as per the $\mathrm{MoH}$ advice.

At NHK, there was already some ICT infrastructure, including implemented LAN and the PIM systems. However, implementation of clinic and pharmacy modules of HIMS at NHK encountered few challenges, and a strategical approach had to be adopted to overcome the following.
1. Staff training
2. Technical issues
3. Inadequacy of resources
4. Change management

\section{Staff training}

Since this was an exigent scheme put into operation in a minimal time frame, formal training was not possible. An unstructured training was provided along with the ongoing work. Some staff members were under the impression that they lacked adequate training and considered that an impediment to this novel system implementation. Nevertheless, most of the staff members realised the importance of training, despite the limited time and resources.

\section{Technical issues}

HIMS clinic and pharmacy module implementation met with some unexpected challenges, most of which inevitably involved system configurations. There were bugs and errors in the system, mostly in the pharmacy module, and the HIMS development team promptly addressed those.

\section{Inadequacy of resources}

Initially, NHK did not possess adequate hardware and maintenance support for system implementation. Within a short time, the hospital was able to acquire new Point of Sale (POS) systems and printers to the pharmacy. The clinics still faced a shortage of computers, and in a short duration, they were provided with the necessary hardware. Since there were no technical team stationed inside the hospital for system support, it was difficult to address the problems that arose while the new system was being implemented. However, the administrative support and commitment of the motivated staff were crucial in overcoming above challenges.

\section{Change management}

Staff resistance to the new clinic system implementation was a prominent obstacle that was identified during the process. It was mainly due to lack of training, communication problems, resistance to change and negative perception about technology. Initially, the staff believed the system would be an additional burden over their usual workload. After the initial phase, the staff apprehended how the process improvement and automation assist the traditional workflows. Since they were used to the paper-based manual system, it was difficult for them to change to a new electronic system abruptly. However, along with some resistance there emerged a new set of champions who assisted the system implementation at large. Those medical officers and pharmacists played a leading role in implementing the system, encouraged the other users to look beyond and prompted them to envisage of the long-term benefits of the electronic system.

The main advantages were process improvement and efficiency, availability of accurate and complete information and quick access to patient information for medical officers and pharmacists, which helped immensely to achieve the objectives of this project. 
Routine feedback was taken, and most users were satisfied with the system.

\section{CONCLUSION}

Despite the challenges, $90 \%$ of NCD clinic care recipients were registered to the system, electronic prescriptions issued, and medications delivered to them.

PIM systems also gained recognition during the COVID-19 pandemic as a vital tool for facilitating clinic medication delivery. The requirement of a single electronic system or need of ensuring interoperability was recognised both by clinicians and administrators. Also, the requirement of a proper mechanism for technical support and maintenance was recognised.

Delivery of medication for NCD clinic care recipients of NHK was carried out by the Department of Posts - Sri Lanka and the extended curative care network of the DoHS-CP. This demonstrated that during COVID-19 pandemic, institutions under three different government sector organisations of the Central Province of Sri Lanka were able to collaborate and adapt to the unexpected situation to cater to urgent needs of the citizens.

\section{REFERENCES}

1. Coronavirus disease (COVID-19) outbreak [Internet]. [cited 2020 May 8]. Available from: https://www.who.int/westernpacific/emergencies /covid-19

2. Coronavirus disease 2019 (COVID-19) Situation Report - 73 [Internet]. World Health Organization; 2020 [cited 2020 May 16]. Available from: https://www.who.int/docs/default-source/corona viruse/situation-reports/20200402-sitrep-73covid-19.pdf?sfvrsn=5ae25bc7_2

3. COVID-19 pandemic in Sri Lanka. In: Wikipedia [Internet]. 2020 [cited 2020 May 5]. Available from: https://en.wikipedia.org/w/index.php?title=COVID -19_pandemic_in_Sri_Lanka\&oldid=954960552

4. Epidemiology Unit [Internet]. [cited 2020 May 10]. Available from: http://www.epid.gov.lk/web /index.php?lang=en

5. Van Dyk L. A Review of Telehealth Service Implementation Frameworks. International Journal of Environmental Research and Public Health. 2014;11(2):1279-1298.

6. Sood S, Mbarika V, Jugoo S, Dookhy R, Doarn CR, Prakash $\mathrm{N}$, et al. What is telemedicine? A collection of 104 peer-reviewed perspectives and theoretical underpinnings. Telemedicine Journal and E-Health: The Official Journal of the American Telemedicine Association. 2007;13(5):573-590.

7. Voran D. Telemedicine and Beyond. Missouri Medicine. 2015;112(2):129-135.

8. Monthly Health Bulletin Feb 2020 - National Hospital Kandy. National Hospital Kandy; 2020.

9. Supply of Pharmaceuticals for Clinic Patients during Curfew period [Internet]. Director General of Health
Services; 2020 [cited 2020 May 27]. Available from: http://www.health.gov.lk/moh_final/english/publi c/elfinder/files/images/2020/Supply\%20of\%20dr ugs $\% 20$ during\%20curfew\%20-

$\% 20 \% 20$ Corrected $\% 20(3) . p d f$

10. Digital Health in Sri Lanka 1998 - 2017 [Internet]. Health Informatics Society of Sri Lanka; [cited 2019 Mar 13]. Available from: http://www.hissl.lk/Digital _Health_SL_1998-2017.pdf

11. National eHealth Guidelines and Standards V 1.0 [Internet]. Ministry of Health; 2016 [cited 2020 May 30]. Available from: http://www.health.gov.lk /moh_final/english/public/elfinder/files/publicati ons/list_publi/NeGS_v_1.pdf 